\title{
Sur la distribution des quantités commensurables.
}

\author{
(Par Ensest Cesiro, étudiant, à Torre Annunziata.)
}

1. La question que nous nous proposons de résoudre ne présenterait aucune difficulté, si nous ne voulions considérer les valeurs commensurables qu'à un point de vue entièrement abstrait, c'est-à-dire sans tenir compte de la manière dont elles sont engendrées. Supposons, en effet, que, ayant une valeur commensurable $z$, il faille ajouter $d z$, nécessairement commensurable, pour obtenir la valeur commensurable, immédiatement supérieure. Il est clair que: $1^{\circ} z+d z$ sera commensurable; $2^{\circ}$ entre $z+d z$ et $z+2 d z$ il n'y aura pas de valeurs commensurables; car s'il y en avait une, en en retranchant $d z$ on obtiendrait une valeur commensurable, comprise entre $z$ et $z+d z$, ce qui est contraire à l'hypothèse. La distribution des quantités commensurables, considérées indépendamment de leur génération, est donc uniforme. Mais l'on peut demander que l'on tienne compte de la manière dont ces valeurs ont été engendrées. Imaginons, par exemple, que, ayant porté, sur une droite indéfinie, à partir d'un point fixe, et dans le même sens, toutes les longueurs entières, on divise chaque intervalle en $n$ parties égales, et supposons que l'on attribue successivement à $n$ les valeurs $2,3,4, \ldots$, jusqu'à l'infini. On vient ainsi à représenter, sur la droite, toutes les quantités commensurables. Il est clair que, dans chaque intervalle, la distribution se reproduit d'une manière identique: il suffit done de rechercher ce qui se passe dans l'intervalle $\overline{01}$. Or, par la division de cet intervalle en $n$ parties égales, après les divisions successives en $2,3, \ldots$; $n-1$ parties, les seuls points nowveaux que l'on introduit correspondent aux $\varphi(n)$ fractions irréductibles, de dénominateur $n$, inférieures à l' unité. Le nombre total des points de division devient donc

$$
\varphi(2)+\varphi(3)+\varphi(4)+\cdots+\varphi(n)=\frac{3}{\pi^{3}} n^{2}
$$


Cela étant, prenons, entre 0 et 1 , une quantité $\varepsilon$, si petite qu'on le veut, mais fixe et differente de zéro. Si $\varphi\left(n, n^{\prime}\right)$ est le nombre des entiers, premiers avec $n$ et non supérieurs à $n^{\prime}$, il est clair que, parmi les $\varphi(n)$ fractions irréductibles, considérées plus haut, il y en a $\varphi(n, n \varepsilon)$ non supérieures $\grave{a} s$. Or, on sait que

$$
\varphi(n, n \varepsilon)=\left[\frac{n \varepsilon}{a}\right] \mu(a)+\left[\frac{n \varepsilon}{b}\right] \mu(b)+\left[\frac{n \varepsilon}{c}\right] \mu(c)+\cdots,
$$

$a, b, c, \ldots$ étant tous les diviseurs de $n$. Par suite, si l'on pose

$$
f(x)=[\varepsilon]+[2 \varepsilon]+[3 \varepsilon]+\cdots+[x \varepsilon],
$$

le nombre des points de division, compris dans l'intervalle $\overline{0 \varepsilon}$, est

$$
\sum_{p=2}^{p=n} \varphi(p, p \varepsilon)=\mu(1) f\left(q_{1}\right)+\mu(2) f\left(q_{2}\right)+\mu(3) f\left(q_{3}\right)+\cdots
$$

Or, on peut écrire

$$
f(x)=\frac{\varepsilon}{2} x^{2}
$$

en négligeant les quantités de l'ordre de $x$. On en déduit, comme d'habitude, l'égalité asymptotique

$$
\sum_{p=2}^{p=n} \varphi(p, p \varepsilon)=\frac{\varepsilon}{2} n^{2} \sum_{ \pm}^{\infty} \frac{\mu(p)}{p^{2}}=\varepsilon \cdot \frac{3}{\pi^{2}} n^{2},
$$

où la quantité négligée n'est pas d'un ordre supérieur à celui de $n \log n$. La probabilité qu'un point de division tombe dans l'intervalle $\overline{0 \varepsilon}$ est donc $\varepsilon$. Par conséquent, la densité des valeurs commensurables est constante dans tout l' intervalle $\overline{01}$, les points extrêmes exceptés. En effet, les raisonnements qui précèdent ne subsisteraient plus si la quantité $\varepsilon$ était nulle ou indéfiniment petite; si, par exemple, elle tendait à zéro de manière que $\varepsilon n=1$. Pour $\varepsilon=0$, remarquons que le point de division le plus proche de 0 est $\frac{1}{n}$. Après $\frac{1}{n}$, il vient, certainement, le point $\frac{1}{n-1}$, séparé de $\frac{1}{n}$ par un intervalle de l'ordre de $\frac{1}{u^{2}}$, dont le rapport à l'intervalle $\overline{0 \frac{1}{n}}$ tend, par conséquent, vers zéro. En conséquence, si la densité est représentée par 1 dans tout un intervalle, elle est 0 aux points extrêmes, mais seulement en ces points; car, si petit que l'on prenne $\varepsilon$, du moment que l'on fixe sa valeur, la probabilité qu'un point de division tombe à l'intérieur de $\overline{0} \bar{\varepsilon}$ ne cesse d'être $\varepsilon$. En d'autres termes, la densité se prísente jci sous forme d'une fonction, admettant une discontinuité ordinaire 
pour toutes les valeurs entières de la variable. Mais c'est exclusivement au point de vue arithmétique que nous voulons étudier la distribution des quantités commensurables. Aussi, dans tout ce que nous allons dire, nous supposerons constamment que ces quantités soient engendrées par la division de deux entiers, pris au hasard.

2. Dans notre article "Sur les éventualités de la division arithmétique", nous avons fait voir que, si $L$ et $\Lambda$ sont les fréquences d'une propriété dans les séries

$$
\left.\begin{array}{llll}
f\left(\frac{n}{1}\right), & f\left(\frac{n}{2}\right), & f\left(\frac{n}{3}\right), \ldots & f\left(\frac{n}{n}\right), \\
f\left(\frac{1}{n}\right), & f\left(\frac{2}{n}\right), & f\left(\frac{3}{n}\right), \ldots & f\left(\frac{n}{n}\right),
\end{array}\right\}
$$

la probabilité que, en prenant $x$ et $y$ au hasard, $f\left(\frac{x}{y}\right)$ jouisse de la propriéte considérée, est

$$
P=\frac{L+\Lambda}{2}
$$

Cela étant, soit $h(x)$ une fonction généralement nulle, mais égale à l'unité pour $x=k$. On a $\left(^{*}\right)$ :

pourvu que

$$
\sum_{p=1}^{p=n} h\left(q_{p}\right)=\sum_{p=1}^{p=n} q_{p} g(p)
$$

d'où :

$$
g(1)+g(2)+g(3)+\cdots+g(x)=h(x),
$$

Donc

$$
g(x)=\mid \begin{gathered}
0, \text { en général, } \\
1, \text { pour } x=k, \\
-1, \text { pour } x=k+1
\end{gathered}
$$

$$
\Sigma h\left(q_{p}\right)=q_{k}-q_{k+1} \text {. }
$$

Par conséquent, si $f(x)=[x]$ dans les séries (1), la fréquence de la valeur $k$ dans la première de ces séries est

$$
L=\lim \cdot \frac{1}{n} \mathbf{\Sigma} h\left(q_{p}\right)=\frac{1}{k(k+1)} .
$$

Si $k$ n'est pas nul, la fréquence de $k$ dans la seconde des séries (25) est évidemment $\Lambda=0$. En conséquence: "la probabilité que $k$ soit le quotient par 
defaut, dans une division quelconque, est $\frac{1}{2 k(k+1)} "$. De même, on a

$$
\mathbf{\Sigma} h\left[i\left(\frac{n}{p}\right)\right]=\mathbf{\Sigma} h\left[\frac{n}{p}+\frac{1}{2}\right]-n h(1)=\mathbf{\Sigma}\left[\frac{2 n}{2 p-1}\right] g(p)-n h(1),
$$

d'où, pour $f(x)=i(x)$ dans la première des séries (1),

$$
L=\frac{2}{2 k-1}-\frac{2}{2 k+1}-h(1) \text {. }
$$

D'autre part, $\Lambda=\frac{h(1)}{2}$. En conséquence:

$$
P=\frac{2}{4 k^{2}-1}-\frac{h(1)}{4} \text {. }
$$

Ainsi: "la probabilité que $k$ soit l'entier le plus proche d'une quantité commensurable, prise au hasard, est $\frac{2}{4 k^{2}-1}$, sauf pour $k=1, k=0$. Dans ces cas, la probabilite est $\frac{5}{12}, \frac{1}{4}$ n.

3. D'après ce qui vient d'être dit, il est facile de s'imaginer, sommairement, la distribution des quantités commensurables dans le système des quantités réelles, positives. En réunissant les résultats obtenus, on trouve qu'elle peut être figurée comme ci-après:

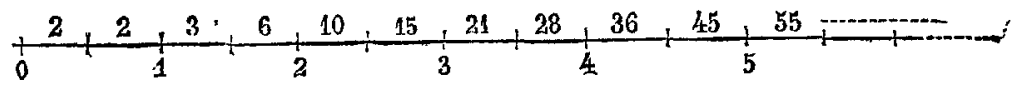

Les nombres écrits à côté des demi-intervalles sont, à partir du troisième, les nombres triangulaires. Ils sont inversement proportionnels aux nombres des quantités commensurables, comprises dans les demi-intervalles respectifs. Il est bon de rappeler jci, une fois pour toutes, que nous considérons les quantités commensurables comme obtenues en prenant, au hasard, deux entiers, et en les divisant l'un par l'autre.

4. Pour compléter l'étude de la distribution, qui nous occupe, cherchons d'abord la probabilité qu' une quantité commensurable quelconque soit comprise entre $k-\varepsilon$ et $k-\varepsilon+1, \varepsilon$ étant une fraction proprement dite, et $k$ un nombre entier. Il suffit de faire $f(x)=[x+\varepsilon]$ dans les séries (1), en continuant à considérer les mêmes fonctions $g$ et $h$. On trouve sans peine

$$
\sum_{1}^{n} h\left[\frac{n}{p}+\varepsilon\right]=\mathbf{\Sigma} h\left[\frac{n}{p}+\varepsilon\right]-\frac{n \varepsilon}{1-\varepsilon} h(1)=\mathbf{\Sigma}\left[\frac{n}{p-\varepsilon}\right] g(p)-\frac{n \varepsilon}{1-\varepsilon} h(1),
$$


d'où, en posant $k-\varepsilon=z$,

$$
L=\frac{1}{z}-\frac{1}{z+1}-\frac{\varepsilon}{1-\varepsilon} h(1) .
$$

D'autre part $\Lambda=\varepsilon h(1)$. En conséquence:

$$
P=\frac{1}{2 z(z+1)}-\frac{\varepsilon^{2}}{1-\varepsilon} \cdot \frac{h(1)}{2} .
$$

Telle est la prubabilité qu'une quantité commensurable quelconque soit comprise entre $z$ et $z+1$. La probabilité qu'elle ne surpasse pas $z$ est donc

$$
\Pi(z)=1-\frac{1}{2 z},
$$

pourvu que $z$ ne soit pas inférieur à 1 . Enfin, la probabilité qu'elle soit comprise entre $z$ et $z+d z$ est donnée par

$$
\Pi(z+d z)-\Pi(z)=\frac{d z}{2 z^{2}} .
$$

On peut énoncer ce résultat en disant que: "La densité des quantités commensurables, autour de $z$, est inversement proportionnelle à $z^{2} n$. En représentant par $D(z)$ une fonction proportionnelle à la densité des quantités commensurables, autour de $z$, on peut done écrire

$$
D(z)=\frac{1}{z^{2}}
$$

Dans le cas de $z<1$, on trouve immédiatement

$$
\Pi(z)=\frac{z}{2}, \quad D(z)=1
$$

La variation des probabilités et des densités peut être représentée par les diagrammes suivants:
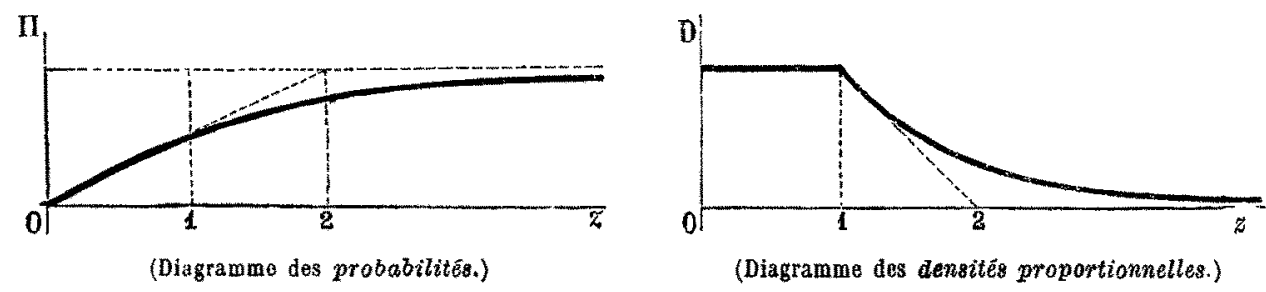

5 . Nous avons tenu à montrer que les propositions précédentes pouvaient être déduites des formules démontrées dans la Note: Sur les éventualités de la 
division arithmétique; mais il est possible de les établir simplement, par une voie toute différente. Dans ce but, prenons, au hasard, deux entiers $x$ et $y$. Nous supposerons, d'abord, que $x$ soit pris parmi les nombres entiers compris entre 0 et $n$, et nous devrons alors adopter pour $y$ le même champ de varia-

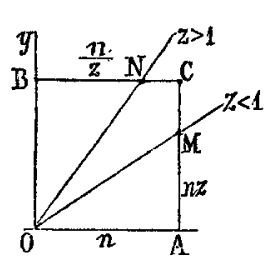
tion. Nous ferons, ensuite, augmenter $n$ indéfiniment. Or, si l'on considère $x$ et $y$ comme les coordonnées d'un point du plan, prendre au hasard $x$ et $y$ c'est prendre au hasard un point à coordonnées entières parmi tous les points d'un carré de côté $n$, indéfiniment grand. La distribution des points à coordonnées entières étant uniforme dans le plan, il est clair que le rapport entre les nombres de ces points, compris à l'intérieur de denx aires indéfiniment grandes est le même que le rapport des deux aires. Par suite, si l'on veut, par exemple, la probabilité que $\frac{y}{x}$ soit moindre que $z,(z<1)$, le problème se réduit à chercher le rapport de l'aire du triangle $O A M$, comprenant les points favorables, à l'aire totale du carré. Si $z>1$, l'aire favorable est celle du trapèze $O A C N$. On retrouve ainsi les formules

$$
\Pi(z)=\frac{z}{2}, \quad \Pi(z)=1-\frac{1}{2 z},
$$

et les densités proportionelles seront, si l'on veut,

$$
D(z)=1, \quad D(z)=\frac{1}{z^{2}}
$$

6. Chaque quantité commensurable $z$, obtenue par la division de deux entiers, pris au hasard, peut être représentée, sur une droite, par un point, situé à la distance $z$ d'un point fixe de la droite. Remarquons que, inversement, tout point de celle-ci représente une infinité de quantités commensurables, ou n'en représente aucune. Ayant, pour airsi dire, condensée sur une droite la représentation des quantités commensurables, nous pouvons, d'une manière analogue, passer à l'étude des systèmes de deux quantités commensurables. Pour abrćger le discours, nous dirons que le nombre complexe $z=x+y \sqrt{-1}$ est commensurable, lorsque $x$ et $y$ sont commensurables. Quelle est, dès lors, la distribution des nombres commensurables dans le plan? Il est clair que la densité de ces nombres, autour de $z$, c'est-à-dire dans le rectangle infinitésimal $d x d y$, est $D(x) D(y)$. Elle est donc inversement proportionnelle aux carrés des coordonnées, supérieures à l'unité. Ayant conduit les parallèles aux axes, à la distance 1 , on partage la portion du plan, contenant les points à coordonnćes 
positives, en quatre régions, comme le montre la figure ci-contre. Dans la région limitée, la densité est 1: dans les régions illimitées suivant un seul axe, elle est représentée par l'inverse du carré de la coordonnée correspondante à cet axe. Enfin, dans la région doublement illimitée, la densité est $\frac{1}{x^{2} y^{2}}$. Nous arons tracé, sur la même figure, des lignes isobariques, ou lieux des points à densité constante: elles sont constituées par des arcs d'hyperboles équilatères, asymptotiques aux axes, dans la région doublement illimitée, et par des

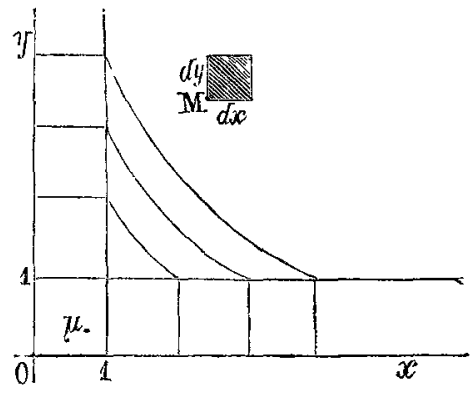
segments rectilignes, parallèles aux axes, dans les deux régions simplement illimitées. Quant à la région limitée, elle est partout isobarique.

7. Le principe précédemment employé pour la recherche directe des formules (3) peut encore s'appliquer ici, et même à des aires finies, parceque la quotité des nombres commensurables, contenus dans une aire finie, est infinie: seulement, il faut tenir compte de la densité. Dans ce but, à un point quelconque $M$ faisons correspondre un point $\mu$, dont chaque coordonnée soit directement ou inversement égale à la coordonnée correspondante de $M$, suivant que celle-ci est inférieure ou supérieure à l'unité. De cette façon, le point $\mu$ est toujours situé dans la région limitée. Il est facile de s'assurer que le rapport des éléments superficiels, pris autour de $\mu$ et de $M$, est proportionnel à la densité des quantités commensurables, autour de $M$. Si $M$ et $\mu$ décrivent des aires $S$ et $\sigma$, la probabilité qu' un nombre commensurable, pris au hasard, soit contenu par $S$ est $\frac{1}{4} \sigma$. En vertu de cette transformation nous pouvons, étant donnée une aire $S$ quelconque, en rapprocher les points commensurables, jusqu'à ce que la densité devienne 1. En d'autres termes, nous pouvons rendre isobarique toute aire qui ne l'est pas. Observons que chaque région a pour transformée la région isobarique, d'où il résulte qu'un nombre commensurable pent tomber, avec une égale probabilité, dans une quelconque des quatre régions, ce qui est évident.

8. Les considérations qui précèdent nous permettent de trouver la probabilité pour qu'une fonction quelconque de deux quantités commensurables, prises au hasard, satisfasse à une certaine condition. Supposons, par exemple, qu' un nombre commensurable, pris au hasard, doive tomber a l'intérieur de l'aire limitée par les axes et par la ligne $A B C D$, figurée ci-après (fig. 1). 
Pour fixer les idées, nous supposerons que la ligne ait la forme générale, indiquée par la figure, et qu'elle soit symétrique par rapport aux axes, de sorte que son équation est, indifféremment, $y=f(x)$ ou $x=f(y)$. La ligne $A B C D$ se transforme en $\alpha \beta \gamma \delta$, et il est clair que les figures $L C D, L M A C, L N B D$,

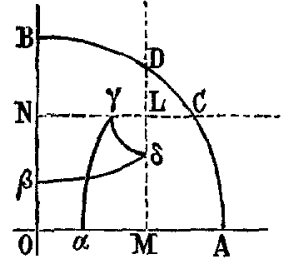

(Fig. 1.)

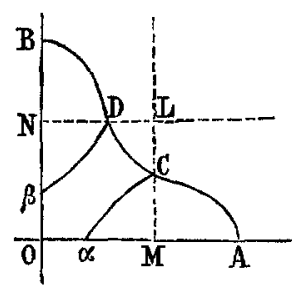

(Fig. 2.)

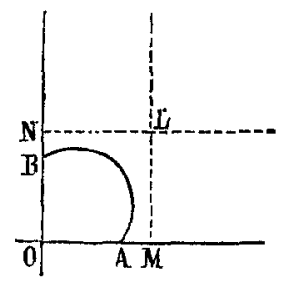

(Fig. 3.)

$O L M N$, qui constituent $O A B C D$, se transforment respectivement en $L \gamma \delta$, $L M \alpha \gamma, L N \beta \delta$, $O L M N$. La moyenne arithmétique des aires de ces dernières figures nous donnera la probabilité cherchée. On trouve, moyennant quelques transformations simples,

$$
P=1-\frac{1}{4 f(1)}-\frac{1}{2} \int_{0}^{4} \frac{d x}{f(x)}-\frac{1}{4} \int_{1}^{f(1)} \frac{d x}{x^{2} f(x)} .
$$

Lorsque $A B C D$ ne traverse pas la région doublement illimitée, c'est-à-djre si elle se trouve dans un des cas indiqués par les fig. 2 et 3 , on obtient ces deux autres formules:

$$
\begin{aligned}
& P=\frac{3}{4} f(1)-\frac{1}{4} \int_{1}^{f(1)} f(x) d x-\frac{1}{2} \int_{0}^{f(1)} \frac{d x}{f(x)}, \\
& P=\frac{1}{4} \int_{0}^{f(0)} f(x) d x .
\end{aligned}
$$

Les deux premières donnent la valeur remarquable

$$
P=\frac{3}{4}-\frac{1}{2} \int_{0}^{1} \frac{d x}{f(x)},
$$

dans le cas où la ligne passe per $L$. Par exemple, loreque la ligne est l'hyperbole équilatère représentée par $x y=1$, on a $f(x)=\frac{1}{x}$; puis la formule (7) donne $P=\frac{1}{2}$, ce qui doit être, comme on peut le voir a priori. Plus généralement, si l'équation de $A B C D$ est $x y=\lambda^{2}$, les formules (4), (5), donnent

$$
P_{\lambda}=1-\frac{1+\log \lambda}{2 \lambda^{2}}, \quad(\lambda>1) ; \quad P_{\lambda}=(1-\log \lambda) \frac{\lambda^{2}}{2}, \quad(\lambda \equiv 1) .
$$


Telles sont les expressions de la probabilité que, ayant pris, au hasard, deux quantités commensurables, leur produit soit inférieur à $\lambda^{2}$. Si $A B C D$ est une droite, de sorte que $f(x)=\lambda-x$, on obtient

$$
\left.\begin{array}{c}
P_{\lambda}=1-\frac{1}{4(\lambda-1)}-\log \sqrt{\lambda}+\left(1+\frac{1}{\lambda^{2}}\right) \log \sqrt{\lambda-1}, \quad(\lambda \geqq 2) ; \\
P_{\lambda}=-\frac{1}{8} \lambda^{2}+\lambda-\frac{3}{4}-\log \sqrt{\lambda}, \quad(2 \equiv \lambda \geqq 1) ; \quad P_{\lambda}=\frac{1}{8} \lambda^{2}, \quad(1 \equiv \lambda) .
\end{array}\right\}
$$

Telles sont les expressions de la probabilité que la somme de deux quantités commensurables, prises au hasard, soit moindre que $\lambda$. Les deux premières formules concordent pour $\lambda=2$; les deux dernières, pour $\lambda=1$. Elles donnent:

$$
P_{2}=\frac{1}{4}(3-\log 4)=0,4034 \ldots ; \quad P_{1}=\frac{1}{8}=0,125 .
$$

Comme dernier exemple, soit $f(x)=\sqrt{\lambda^{2}-x^{2}}$. Si l'on pose $\sin \omega=\frac{1}{\lambda}$, on trouve

$$
\begin{aligned}
& P=1-\frac{1}{2}(\omega+\sin \omega \cos \omega), \quad(\lambda \geqq \sqrt{2}) ; \\
& P=\frac{4}{16}\left\{(4 \omega-\pi) \cot ^{2} \omega+12 \cot \omega+12 \omega-5 \pi\right\}, \quad P=\frac{\pi \lambda^{2}}{16},
\end{aligned}
$$

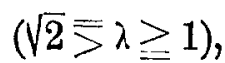

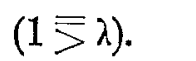

Ainsi, pour $\lambda=\sqrt{2}$, les deux premières formules donnent

$$
P=\frac{3}{4}-\frac{\pi}{8},
$$

c'est-à-dire que: “Il y a 9 à parier, contre 5 environ, que la somme des carrés de deux quantités commensurables, prises au hasard, est supérieure à 2 n.

9. À leur tour, les formules (8), (9), (10), permettent de résoudre une foule d'autres questions de probabilités. Par exemple, d'après (8), la probabilité que $x y$ soit compris entre deux nombres entiers consécutifs, $n$ et $n+1$, est

$$
p_{n}=P_{\sqrt{n+1}}-P_{\sqrt{n}}=\frac{1}{2}\left(\frac{1}{n}-\frac{1}{n+1}\right)+\frac{1}{4}\left(\frac{\log n}{n}-\frac{\operatorname{lng}(n+1)}{n+1}\right) .
$$

Il en résulte

$$
p_{1}+p_{3}+p_{5}+\cdots=\log \sqrt{2}+\frac{s}{4},
$$

en désignant par $s$ la somme de la série

$$
\frac{\log 1}{i}-\frac{\log 2}{2}+\frac{\log 3}{3}-\frac{\log 4}{4}+\cdots
$$


Cela étant, il est clair que, si l'on fait

$$
S_{n}=\frac{\log 1}{1}+\frac{\log 2}{2}+\frac{\log 3}{3}+\cdots+\frac{\log n}{n},
$$

on a, en supposant $n$ pair,

$$
s_{n}=S_{n}-\left(\frac{\log 2}{1}+\frac{\log 4}{2}+\cdots+\frac{\operatorname{lng} n}{\frac{n}{2}}\right)=S_{n}-S_{\frac{n}{2}}-H\left(\frac{n}{2}\right) \cdot \log 2 .
$$

Or, on sait que

$$
S_{n}=A+\frac{1}{2}(\log n)^{2}+R_{n},
$$

$A$ étant une constante, et $R_{n}$ une fonction de $n$, qui tend à zéro pour $n$ infini. On en déduit

$$
s_{n}=\frac{1}{2}(\log 2)^{2}-\left\{H\left(\frac{n}{2}\right)-\log \frac{n}{2}\right\} \log 2+R_{n}-R_{\frac{n}{2}}
$$

puis, pour $n$ infini,

$$
s=\frac{1}{2}(\log 2)^{2}-C \log 2 .
$$

Conséquemment: "la probabilité que le plus grand nombre entier, contenu dans le produit de deux quantités commensurables, prises au hasard, soit impair, est

$$
\frac{1}{2}(\log \sqrt{2}+2-C) \log \sqrt{2}=0,30660 \ldots " .
$$

10. Avec la même facilité on résout les ouestions relatives à des systèmes de trois quantitćs commensurables. Si l'on veut savoir, par exemple,

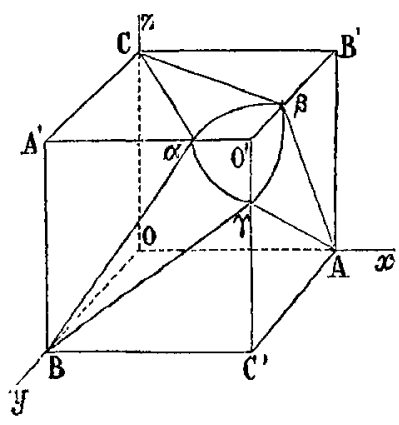
quelle probabilité il y a pour que le produit de trois quantités commensurables, prises au hasard, soit inférieur à une quantité donnée $\lambda^{3}$, on ramène immédiatement la question à la cubature de certains volumes. Soit, pour fixer les idées, $\lambda>1$. La surface représentée par $x y z=\lambda^{3}$ traverse sept des huit régions de l'espace, qu'il y a lieu de considérer. Le volume compris entre cette surface et les plans coordonnés se trouve divisé en huit parties. Une d'elles est la région isobarique, dont le volume est 1. La partie limitée par la portion de la surface, qui se trouve dans la région triplement illimitée, se transforme en un volume $0^{\prime} \alpha \beta \%$, que nous appellerons $u$. Un 
calcul simple donne

$$
u=1-\frac{2+6 \log \lambda+9(\log \lambda)^{2}}{2 \lambda^{3}} .
$$

Les portions de surface, contenues dans les trois régions doublement illimitées, se transforment dans les paraboloildes hyperboliques $B C \beta \gamma, C A \gamma_{\alpha}, A B \alpha \beta$, limitant, dans le cube isobarique, du côté des arêtes $O^{\prime} A^{\prime}, O^{\prime} B^{\prime}, O^{\prime} C^{\prime}$, trois volumes égaux à $v$. On troure

$$
v=1-\frac{3}{4} \cdot \frac{1+2 \log \lambda}{\lambda^{3}} .
$$

Enfin, les portions de surface, contenues dans les régions simplement illimitées, ont pour transformées les paraboloïdes hyperboliques $B C \alpha, C A \beta, A B \gamma$, limitant, du côté des arêtes $O A, O B, O C$, trois volumes isobariques, égaux à $w$. On trouve

$$
w=1-\frac{1}{4 \lambda^{3}} .
$$

Done:

$$
P=\frac{1}{8}(1+3 v+3 w+u)=1-\frac{8+15 \log \lambda+9(\log \lambda)^{2}}{16 \lambda^{3}} .
$$

Par exemple: "La probabilité que le produit de trois quantités commensurables, prises au hasard, surpasse le nombre e, est $\frac{2}{13}$.

11. En général, on peut étudier ces questions dans un espace à $k$ dimensions, dont on partage la partie positive en régions, moyennant les plans $x_{1}=1, x_{2}=1, \ldots x_{k}=1$, de sorte que, dans une région $r$ fois illimitée, $r$ des coordonnées d'un point quelconque sont supérieures à l'unité. Le nombre de ces régions est $C_{k, r}$, et le nombre total des régions est $2^{k}$. La densité des nombres, $(k-1$ fois complexes), commensurables, varie toujours en raison inverse du produit carré des coordonnées supérieures à l'unité. Cette idée des transformations arithmétiques des espaces mèrite d'être amplement développée. Outre ses applications à des questions de probabilités et à des questions de moyennes, qui se ramènent toujours à des déterminations barycentriques, elle est surtout utile pour l'étude des distributions, qu'il s'agisse ou non de quantités commensurables. Pour cela, elle doit s'aider du principe de la condensation des espaces. La droite sur laquelle nous avons commencé à étudier les quantités commensurables nous a offert un premier exemple de condensation. Nous en avons déduit des espaces à densité variable, et, en particulier, un plan, qui est la condensation d'une figure d'un espace à quatre dimensions. 
Ce plan pourra subir une nouvelle condensation, et ainsi de suite. L'utilité de ces condensations est visible. D'abord des questions, relatives à des espaces inabordables par la pensée, sont reconduites dans les espaces réels. D'autre part, les quantités arithmétiques, représentées géométriquement, sont répandues d'une manière discontinue, de sorte quon ne peut leur appliquer les formules $d u$

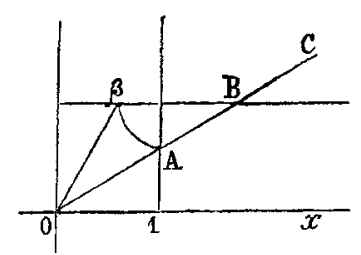

Calcul intégral. Après condensation, cet inconvénient disparaît, car les points représentatifs se rapprochent, coïncident, et créent ainsi un espace à densité variable, non continu, mais à discontinuités infinitésimales. Pour montrer un exemple de condensations successives, reprenons le plan, sur lequel nous avons représenté les nombres complexes commensurables. Si $x$ et $y$ sont deux quantités commensurables, prises au hasard, pour calculer la probabilité que $\frac{y}{x}$ soit moindre que $z$, il faut transformer d'abord la droite $O A C$, de manière à rendre isobarique l'aire $C O x$. On voit immédiatement que $O A, A B, B C$ se transforment en $O A, A \beta$, $\beta O$. Par le calcul habituel on trouve

$$
\Pi(z)=\frac{z}{4}(2-\log z) \quad(z<1) .
$$

Si $z>1$, il est clair que $\Pi(z)$ s'obtient en retranchant de 1 l'expression précédente, après y avoir changé $z$ en $\frac{1}{z} \cdot$ Par conséquent

$$
\Pi(z)=1-\frac{2+\log z}{4 z} \quad(z>1)
$$

La représentation étant ainsi condensée sur une droite, la densité en chaque point sera donnée par une des deux formules qui suivent:

$$
D(z)=1-\log z, \quad D(z)=\frac{1+\log z}{z^{2}} .
$$

Par extension au plan, nous pourrons résoudre, avec la plus grande facilité, une foule de questions, relatives à huit nombres entiers, pris au hasard, c'està-dire des questions, qui, par les procédés habituéls, exigeraient une représentation dans un espace à huit dimensions.

12. Dans la formation d'une quantité commensurable, au moyen de deux nombres entiers $x$ et $y$, on a accordé, jusqu'ici, pleine liberté de variation aux deux entiers. Mais il peut se faire que, par la manière même dont on pose la question, les deux champs de variation viennent à se limiter mutuellement. 
Supposons, par exemple, que les deux nombres, au lieu d'être pris tout à fait au hasard, aient été obtenus par décomposition arbitraire, en deux parties entières, d'un nombre entier, pris au hasard. Alors, le champ de variation, au lieu d'être un carré, sera un triangle rectangle, isoscèle, de côtés $n$ indéfiniment grands. On voit que la probabilité d'avoir $\frac{y}{x}<z$ a pour expression unique

Par suite

$$
\Pi(z)=\frac{z}{1+z}
$$

$$
D(z)=\frac{1}{(1+z)^{2}}
$$

Si l'on étend la représentation au plan, on voit que la densité en un point quelconque est l'inverse de $(1+x)^{2}(1+y)^{2}$. La transformation à employer, pour rendre isobarique une aire donnée, est définie par les relations

$$
x^{\prime}=\frac{1}{1+x}, \quad y^{\prime}=\frac{1}{1+y} .
$$

Ici aussi toutes les figures transformées sont contenues par un carré de côté 1. $\mathrm{Si} \sigma$ est la transformée de $S$, la probabilité qu'un point tombe à l'intérieur de $S$ est $\sigma$. Les applications seraient faciles: nous les omettons.

13. Dans une Note précédente, nous avons rencontré certaines questions, que les formules ordinaires, dans leur état actuel, ont été impuissantes à résoudre. Il n'en est pas ainsi de la représentation géométrique. Celle-ci nous fournira, plus tard, les moyens d'approximation qui nous manquent. Pour le moment, nous l'appliquerons à la résolution de quelques questions de moyennes, en faisant observer que la comparaison des résultats que nous allons obtenir, avec les formules écrites dans les Notes précédentes, conduit à des déterminations asymptotiques importantes, dont nous parlerons une autre fois. D'abord, si la valeur moyenne de $f(z)$ est constante, elle sera évidemment donnée par

$$
\mathfrak{n}=\frac{\int_{0}^{\infty} f^{\prime}(z) D(z) d z}{\int_{0}^{\infty} D(z) d z} .
$$

Lorsque $z$ est une quantité commensurable, prise au hasard, cette formule devient

$$
\mathfrak{m}=\frac{1}{2} \int_{0}^{1} f(z) d z+\frac{1}{2} \int_{1}^{\infty} f(z) \frac{d z}{z^{z}} .
$$


Si l'on veut, par exemple, la moyenne valeur de $z-[z]$, on a

$$
\mathfrak{A}=\frac{1}{2} \int_{0}^{1} z d z+\frac{1}{2} \sum_{q=1}^{q=\infty} \int_{q}^{q+1}(z-q) \frac{d z}{z^{2}}
$$

ou bien

$$
\mathfrak{A}=\frac{1}{4}+\frac{1}{2} \sum_{1}^{\infty}\left\{\log \left(1+\frac{1}{q}\right)-\frac{1}{q+1}\right\}=\frac{3}{4}-\frac{C}{2},
$$

conformément à ce qui a été trouvé dans une Note antérieure. De même, pour $f(z)=z-i(z)$, on a d'abord

$$
\mathfrak{A}=\frac{1}{2} \int_{0}^{\frac{1}{2}} z d z+\frac{1}{2} \int_{\frac{1}{2}}^{1}(z-1) d z-\frac{1}{2} \int_{\frac{1}{2}}^{1}(z-1) \frac{d z}{z^{2}}+\frac{1}{2} \sum_{q=1}^{q=\infty} \int_{q-\frac{1}{2}}^{q+\frac{1}{2}}(z-q) \frac{d z}{z^{2}}
$$

puis:

$$
\mathfrak{H}=\frac{1}{2}(1-\log 2)+\frac{1}{2} \sum_{1}^{\infty}\left\{\log \frac{2 q+1}{2 q-1}-\left(\frac{1}{2 q-1}+\frac{1}{2 q+1}\right)\right\}=1-\log 2-\frac{C}{2},
$$

résultat connu. Venons, maintenant, à un problème qui nous intéresse, et supposons que $f(z)$ soit la valeur absolue de la différence $z-i(z)$. On voit aisément que

$$
\begin{aligned}
\mathfrak{A l} & =\frac{1}{2} \int_{0}^{1} z d z+\frac{1}{2} \int_{\frac{1}{2}}^{1}(1-z) d z-\frac{1}{2} \int_{\frac{1}{2}}^{1}(1-z) \frac{d z}{z^{2}}+ \\
& +\frac{1}{2} \sum_{q=1}^{q=\infty}\left\{\int_{q-\frac{1}{2}}^{q}(q-z) \frac{d z}{z^{2}}+\int_{q}^{q+\frac{1}{2}}(z-q) \frac{d z}{z^{2}}\right\},
\end{aligned}
$$

c'est-à-dire:

$$
\mathbf{m}=\frac{1}{8}-\frac{1}{2}(1-\log 2)+\frac{1}{2} \underset{1}{\mathbf{y}}\left\{\log \frac{2 q-1}{2 q} \cdot \frac{2 q+1}{2 q}+\left(\frac{1}{2 q-1}-\frac{1}{2 q+1}\right)\right\},
$$

d'où, en vertu de la formule de Wallis,

$$
\mathfrak{A}=\frac{1}{8}+\log \frac{2}{\sqrt{\pi}}=0,245782 \ldots
$$

Telle est la valeur moyenne de la différence absolue entre une quantité commensurable quelconque et le nombre entier qui s'en rapproche le plus. Tel est aussi, dans une division quelconque, le moyen rapport du plus petit reste au divisew. 
14. Cherchons la moyenne valeur $r_{m}$ du $m^{\text {ème }}$ chiffre décimal, dans une division quelconque. Il est clair que, dans la formule (12), la somme des intégrales correspondante au chiffre $r$ est le produit de $r$ par

c'est-à-dire:

$$
\sum_{q=1}^{q=10^{m-1}} \int_{\frac{q-1}{10^{m-1}}+\frac{r}{10^{m}}}^{\frac{q-1}{10^{m-1}}+\frac{r+1}{10^{m}}} d z+\sum_{q=1}^{q=\infty} \int_{1+\frac{q-1}{10^{m-1}}+\frac{r}{10^{m}}}^{1+\frac{q-1}{10^{m-1}+\frac{r+1}{10^{m}}}} \frac{d z}{z^{2}}
$$

$$
\frac{r}{10}+10^{m-1} r\left\{H\left(10^{m-1}-1+\frac{r+1}{10}\right)-H\left(10^{m-1}-1+\frac{r}{10}\right)\right\} .
$$

Conséquemment:

$$
r_{m}=\frac{9}{4}+\frac{10^{m-1}}{2}\left\{10 H\left(10^{m-4}\right)-\sum_{p=1}^{p=10} H\left(10^{m-2}-1+\frac{p}{10}\right)\right\},
$$

ou, enfin,

$$
r_{m}=\frac{9}{4}+\frac{10^{m}}{2}\left\{\log 10-H\left(10^{m}\right)+H\left(10^{m-1}\right)\right\} .
$$

Par exemple:

$$
r_{1}=5 \log 10-\frac{3727}{504}=4,1230 \ldots
$$

Telle est la moyenne valeur du premier chiffre décimal, dans une division quelconque. Si $m$ augmente indéfiniment, $r_{m}$ tend vers $4+\frac{1}{2}$.

15. Quelle est la valeur moyenne du dernier chiffre? Évidemment, d'après $(12)$,

ou bien

$$
\mathfrak{n}=\frac{1}{2} \sum_{q=0}^{q=\infty} \sum_{r=1}^{r=q} r \int_{10_{q}+r}^{10 q+r+1} \frac{d z}{z^{2}}
$$

$$
\mathfrak{n}=\frac{1}{2} \sum_{q=1}^{q=\infty}\left(\frac{1}{10 q+1}+\frac{1}{10 q+2}+\cdots+\frac{1}{10 q+10}-\frac{1}{q+1}\right)=\log \sqrt{10} .
$$

Ainsi: " dans une division quelconque, le dernier chiffre du quotient est moyennement égal à $1,15179 \ldots$ " La petitesse du résultat est due à ce que, dans la moitié des divisions, le quotient est zéro.

16. Lorsque $f$ est une fonction indicatrice, l'expression de sert à résoudre des questions de probabilités. Par exemple, la probabilité que $r$ soit le dernier chiffre du quotient est, pour $r>0$,

$$
P_{r}=\frac{1}{2} \sum_{q=0}^{q=\infty} \int_{10_{q}+r}^{10 q+r+1} \frac{d z}{z^{2}}=\frac{1}{20}\left\{H\left(\frac{r+1}{10}-i\right)-H\left(\frac{r}{10}-1\right)\right\}
$$


Pour $r=0$,

On trouve encore

$$
P_{0}=\frac{1}{2}+\frac{1}{20} H\left(\frac{1}{10}\right)
$$

$$
P_{r}+P_{9-r}=\frac{\pi}{20} \cdot \frac{\sin 18^{\circ}}{\sin \frac{\pi r}{10} \cdot \sin \frac{\pi(r+1)}{10}}, \quad(r>0)
$$

et

$$
P_{0}+P_{9}=1-\frac{\pi}{20} \cot 18^{\circ}
$$

Par exemple: a la probabilité que, dans une division quelconque, le dernier chiffre du quotient soit 4 ou 5 , est $\frac{\pi}{20} \operatorname{tg} 18^{\circ} \%$.

17. D'une manière générale, la formule (12) permet d'affirmer que si, ayant pris au hasard deux entiers, on divise le plus grand par le plus petit, la probabilité que le quotient par défaut appartienne à un certain système $\Omega$ est

$$
P=\frac{f(1)}{1 \cdot 2}+\frac{f(2)}{2 \cdot 3}+\frac{f(3)}{3 \cdot 4}+\frac{f(4)}{4 \cdot 5}+\cdots,
$$

$f(x)$ étant 1 ou 0 , suivant que $x$ appartient ou n'appartient pas à $\Omega$. Si, par exemple, $\Omega$ est le système des nombres carrés, on trouve

$$
P=\frac{1}{2}+\frac{\pi^{2}}{6}-\frac{\pi}{2} \cdot \frac{e^{2 \pi}+1}{e^{2 \pi}-1}
$$

Telle est la probabilité que dans la division d'un nombre quelconque, par un nombre plus petit, le quotient soit un carré. De même, la probabilité que, dans la même division, le quotient le plus approché appartienne à $\Omega$ est

$$
P=4\left\{\frac{f(1)}{1 \cdot 3}+\frac{f(2)}{3 \cdot 5}+\frac{f(3)}{5 \cdot 7}+\cdots\right\}-f(1) ;
$$

etc., etc.... Les applications, fort nombreuses, sont trop faciles pour que nous insistions davantage.

18 L'expression de $\mathfrak{m}$, prise entre des limites finies, est destinée à devenir de la plus grande importance dans la théorie des moyennes; car nous l'emploierons à déterminer le premier terme de toute représentation moyenne ou asymptotique. Cependant, il est bon d'observer que la formule (11), sous sa forme actuelle, constitue déjà un moyen de détermination asymptotique, plus délicat que les formules dont nous faisons usage habituellement. Supposons, par exemple, qu'il s'agisse d'évaluer moyennement, jusqu'aux quantités de l'ordre 
de $n$, la somme $\mathbf{\Sigma} q_{p}^{2}$. Une induction fort simple permet d'affirmer que l'expression cherchée a la forme

$$
\sum_{1}^{n} q_{p}^{2}=\frac{\pi^{2}}{6} n^{2}+A n \log n+B n
$$

où $A$ et $B$ sont des constantes à déterminer. D'ailleurs

$$
\sum_{1}^{n} \frac{n^{2}}{p^{2}}=\frac{\pi^{2}}{6} n^{2}-n, \quad \sum_{1}^{n} \frac{n}{p}=n \log n+C n,
$$

en négligeant les quantités d'un ordre inférieur à celui de $n$. En conséquence:

D'autre part

$$
\sum_{1}^{n}\left\{\frac{n^{2}}{p^{2}}+A \frac{n}{p}-q_{p}^{2}\right\}=(A C-B-1) n \text {. }
$$

$$
\sum_{1}^{n}\left\{\frac{p^{2}}{n^{2}}+A \frac{p}{n}-\left[\frac{p}{n}\right]^{2}\right\}=\left(\frac{1}{3}+\frac{A}{2}\right) n .
$$

Par suite, d'après (2),

$$
\mathfrak{M}=\frac{1}{2}\left(\frac{1}{3}+\frac{A}{2}\right)+\frac{1}{2}(A C-B-1) .
$$

pourvu que $f(z)=z^{2}+A z-[z]^{2}$. Donc, en vertu de (12),

$$
\left(\frac{1}{3}+\frac{A}{2}\right)+(A C-B-1)=\int_{0}^{2}\left(z^{2}+A z\right) d z+\sum_{q=1}^{q=\infty} \int_{q}^{q+1}\left(z^{2}+A z-q^{2}\right) \frac{d z}{z^{2}},
$$

ou bien

$$
\begin{aligned}
& A C-B-1=\sum_{q=1}^{q=\infty}\left\{A \log \left(1+\frac{1}{q}\right)+\frac{1}{q+1}\right\}= \\
= & A \sum_{1}^{\infty}\left\{\log \left(1+\frac{1}{q}\right)-\frac{1}{q+1}\right\}+(A+1) \sum_{1}^{\infty} \frac{1}{q+1} .
\end{aligned}
$$

Dans le second membre, la première serie est convergente, et a pour somme $1-C$; mais la seconde est divergente. On doit donc prendre $A=-1$. La dernière relation devient alors $B=-2 C$. Conséquemment

Par combinaison avec

$$
\sum_{1}^{n} q_{p}^{2}=\frac{\pi^{2}}{6} n^{2}-n \log n-2 C n
$$

on trouve

$$
\sum_{1}^{n} q_{p}=n \log n+(2 C-1) n
$$

$$
\sum_{1}^{n} \int p=\Sigma p q_{p}=\frac{1}{2} \mathbf{\Sigma} q_{p}^{2}+\frac{1}{2} \mathbf{\Sigma} q_{p}=\frac{\pi^{2}}{12} n^{2}-\frac{n}{2} .
$$


Donc, en moyenne,

$$
\int x=\frac{\pi^{2}}{6} x-\frac{1}{2}
$$

19. Rappelons-nous aussi que nous n'avons pu obtenir directement la valeur de la constante $A$ dans la somme

$$
\sum_{1}^{n} p q_{p}(-1)^{2\left(\frac{n}{p}\right)}=A n^{2} \text {. }
$$

Maintenant, si l'on fait $f(z)=(-1)^{i(z)} \cdot \frac{[z]}{z}$, dans (12), on obtient immédiatement

c'est-à-dire :

$$
A=\sum_{1}^{\infty}\left\{\int_{q}^{q+\frac{1}{2}}(-1)^{q} q \frac{d z}{z^{3}}+\int_{q+\frac{1}{2}}^{q+1}(-1)^{q+1} q \frac{d z}{z^{3}}\right\}
$$

$$
A=\frac{1}{2} \sum_{1}^{\infty}(-1)^{q} q\left\{\frac{1}{q^{2}}+\frac{1}{(q+1)^{2}}-\frac{8}{(2 q+1)^{2}}\right\}=2 G-\frac{\pi}{2}-\frac{\pi^{2}}{24} .
$$

20. Autre application: $\chi(x)$ étant le logarithme du plus petit commun multiple des $x$ premiers nombres naturels, on sait $\left({ }^{*}\right)$ que, asymptotiquement,

Par suite:

$$
\sum_{1}^{n} \chi\left(q_{p}\right)=n \log n-n
$$

Puis:

$$
\sum_{1}^{n}\left\{\frac{n}{p}-\chi\left(q_{p}\right)\right\}=\left(1+C^{n}\right) n
$$

$$
1+C=\sum_{q=1}^{q=\infty} \int_{q}^{q+1}\{z-\chi(q)\} \frac{d z}{z^{2}}=\sum_{1}^{\infty}\left\{\log \left(1+\frac{1}{q}\right)-\frac{\chi(q)}{q(q+1)}\right\}
$$

d'où, en introduisant la fonction $ノ(x)$, et en observant que, pour $n$ infini, $\frac{\chi(n)}{n}$ tend vers l'unité,

$$
\lim \cdot\left(\log n-\left\{\frac{v(1)}{1}+\frac{v(2)}{2}+\frac{v(3)}{3}+\cdots+\frac{v(n)}{n}\right\}\right)=C,
$$

pour $n$ infini. Sous une autre forme, en désignant par $\varpi_{1}, \varpi_{2}, \varpi_{3}, \ldots \varpi_{\text {g }}$ la série des nombres premiers $2,3,5, \ldots$, non supérieurs à $n$, on peut écrire

$$
\lim \cdot\left\{\log n-\sum_{r=1}^{r=9} \frac{\log \varpi_{r}}{\pi_{r}-1}\right\}=C
$$

résultat important, connu (*). 
21. En changeant le mode de génération des quantités commensurables, l'égalité (11) prend d'autres formes. Ainsi, en employant une génération, à laquelle il a été fait allusion plus haut, on a:

$$
\mathfrak{A t}=\int_{0}^{\infty} \frac{f(z) d z}{(1+z)^{2}} .
$$

Par exemple, soit $f(z)$ égal à 1 ou à 0 , suivant que $i(z)$ est impaìr ou pair. On obtient

$$
\mathfrak{H}=\sum_{1}^{\infty} \int_{2 q-\frac{3}{2}}^{2 q-\frac{1}{2}} \frac{d z}{(1+z)^{2}}=2 \sum_{1}^{\infty}\left(\frac{1}{4 q-1}-\frac{1}{4 q+1}\right)=2-\frac{\pi}{2} .
$$

Donc: "On divise l'une par l'autre les deux parties entières, arbitraires, d'un nombre entier, pris au hasard. La probabilité que le quotient le plus approché soit pair est $0,57079 \ldots "$. tement

Veut-on la moyenne valeur de $z-[z]$ ? La formule (13) donne immédia-

$$
z-[z]=\sum_{1}^{\infty} \int_{q-1}^{q} \frac{z-(q-1)}{(1+z)^{2}} d z=\sum_{1}^{\infty}\left\{\log \left(1+\frac{1}{q}\right)-\frac{1}{q+1}\right\}=1-C .
$$

De même

$$
i(z)-[z]=\sum_{\frac{1}{q}}^{\infty} \int_{q-\frac{1}{2}}^{q} \frac{d z}{(1+z)^{2}}=2 \sum_{1}^{\infty}\left(\frac{1}{2 q+1}-\frac{1}{2 q+2}\right)=\log 4-1 .
$$

Donc: "Lorsqu'on divise l'une par l'autre les deux parties entières, arbitraires, d'un entier, pris au hasard, il y a 27 à parier, contre 17 environ, que le quotient le plus approché est celui par défaut".

(") Voyez Premier Mémoire d'Arithmétique. 